AperTO - Archivio Istituzionale Open Access dell'Università di Torino

\title{
Ceramides and diabetes mellitus: an update on the potential molecular relationships
}

\section{This is a pre print version of the following article:}

Original Citation:

Availability:

This version is available http://hdl.handle.net/2318/1729002

since 2020-02-20T10:43:14Z

Published version:

DOI:10.1111/dme.13943

Terms of use:

Open Access

Anyone can freely access the full text of works made available as "Open Access". Works made available under a Creative Commons license can be used according to the terms and conditions of said license. Use of all other works requires consent of the right holder (author or publisher) if not exempted from copyright protection by the applicable law. 


\section{Ceramides and diabetes mellitus: an update on the potential molecular relationships}

Habib Yaribeygi, ${ }^{1}$ Alexandra E. Butler, ${ }^{2}$ Simona Bo, ${ }^{3}$ Massimiliano Ruscica, ${ }^{4}$ Amirhossein Sahebkar ${ }^{5,6,7^{*}}$

${ }^{1}$ Health Research Center, Life Style Institute, Baqiyatallah University of Medical Sciences, Tehran, Iran

${ }^{2}$ Diabetes Research Center, Qatar Biomedical Research Institute, Doha, Qatar.

${ }^{3}$ Department of Medical Sciences, AOU Città della Salute e della Scienza di Torino, University of Turin, Torino, Italy.

${ }^{4}$ Dipartimento di Scienze Farmacologiche e Biomolecolari, Università degli Studi di Milano, Milan, Italy

${ }^{5}$ Neurogenic Inflammation Research Center, Mashhad University of Medical Sciences, Mashhad, Iran ${ }^{6}$ Biotechnology Research Center, Pharmaceutical Technology Institute, Mashhad University of Medical Sciences, Mashhad, Iran

${ }^{7}$ School of Pharmacy, Mashhad University of Medical Sciences, Mashhad, Iran

*Corresponding Author: Amirhossein Sahebkar, Biotechnology Research Center, Mashhad University of Medical Sciences, Mashhad 9177948564, Iran. Tel: +985138002299; Fax: +985138002287; E-mail: sahebkara@mums.ac.ir; amir_saheb2000@yahoo.com

Running title: Ceramides in diabetes 


\begin{abstract}
Recent evidence suggests that ceramide can play an important pathophysiological role in the development of diabetes. Ceramides are primarily recognized as lipid bilayer building blocks, but recent work has shown that these endogenous molecules are important intracellular signaling mediators and may exert some diabetogenic effects via molecular pathways involved in insulin resistance, beta cell apoptosis, and inflammation. Here, we present available evidence on the possible roles of ceramides in diabetes mellitus and introduce eight different molecular mechanisms mediating the diabetogenic action of ceramides categorized into those predominantly related to insulin resistance versus those mainly implicated in betacell dysfunction. Specifically, the mechanistic evidence involves beta cell apoptosis, pancreatic inflammation, mitochondrial stress, endoplasmic reticulum (ER) stress, adipokine release, IRS-1 (Insulin receptor substrate 1) phosphorylation, oxidative stress and insulin synthesis. The collective evidence suggests that therapeutic agents aimed at reducing ceramide synthesis and lowering circualating levels may be beneficial in prevention and/or treatment of diabetes and its related complications.
\end{abstract}

Keywords: diabetes mellitus, ceramide, adipokines, oxidative stress, insulin resistance 


\section{Novelty Statement}

- Ceramides are building blocks of cellular lipid bilayers and have been causally implicated in the pathophysiology of diabetes

- Recent evidence suggests that ceramides act as intracellular signalling molecules and may exert diabetogenic effects via pathways involved in insulin resistance, beta cell apoptosis and inflammation

- Here, we present the mechanistic evidence for the role of ceramides in diabetes

- New therapeutic agents that lower plasma levels of ceramides may be beneficial in prevention and/or treatment of diabetes and its related complications. 


\section{Introduction}

The global incidence of diabetes mellitus has reached epidemic proportions, and diabetes is now established as the most prevalent metabolic disorder worldwide. The International Diabetes Federation currently estimates there to be 366 million adults with diabetes, representing more than $8 \%$ of the global population, and this is projected to rise to 552 million by the year 2030. Additionally, there is a huge predicted number of undiagnosed cases of diabetes. The vast majority of adults with diabetes have type 2 diabetes, and, thus, the increasing prevalence of diabetes globally is largely due to an increase in type 2 diabetes [1].

Many of the debilitating complications of diabetes appear in middle to older age, and cardiovascular disease, retinopathy and renal failure are strongly related to a history of uncontrolled diabetes. Managing this disorder to maintain tight glycemic control is a key element in the prevention and delay of development of these recognized complications. The exact pathophysiology of diabetes is unknown, though a variety of different factors, such as low-grade chronic inflammation, genetic background and gene defects, pancreatic beta cell failure and insulin resistance in peripheral tissues are each known to play a role. Additionally, sphingolipids are purported to promote cellular conditions conducive to the development of diabetes mellitus [2].

Ceramides are a family of lipid molecules which are present in large amounts in the lipid bilayer membranes of eukaryotic cells [3]. In addition to making the cell membrane hydrophobic, these molecules are also considered to be bioactive agents which participate in multiple intracellular pathways, examples being in the generation of free radicals, the release of inflammatory cytokines, apoptotic processes and modulation of gene expression [4]. Therefore, a role for ceramides has been proposed in cardiovascular and neurodegenerative diseases as well as in cancer, depression, inflammation and obesity [5]. Moreover, recent 
evidence has implicated the involvement of ceramides in failing insulin signaling, insulin resistance and, ultimately, diabetes development [6].

In this review, we consider the evidence for the possible mechanistic role of ceramides in the development of diabetes mellitus and its complications.

\section{The Physiology of Ceramides}

Ceramides are naturally occurring lipid molecules that are fundamental building blocks found in abundance in eukaryotic cell membranes. These physiological lipids are principally composed of sphingosine and fatty acids that are incorporated during sphingomyelin synthesis. They are a major component of the cellular lipid bilayer membrane and have an important role in maintaining its integrity (Figure 1) [7].

Ceramide synthesis occurs in at least three distinct molecular pathways: the de novo pathway, the sphingomyelin hydrolysis (degradation) pathway and the salvage (recycling) pathway (Figure 2) [8].

The de novo pathway occurs most commonly in the endoplasmic reticulum (ER) and is initiated by the rate-limiting enzyme, serine palmitoyl transferase,that produces 3-ketodihydrosphingosine, which in turn is reduced to dihydrosphingosine. Dihydrosphingosine is then converted to ceramide by dihydroceramide synthase and dihydroceramide desaturase enzyme activity [9]. There are six ceramide synthases (CerS1-6), each producing ceramides

with specific chain lengths, functions, tissue distribution, and effects [5]. The highest tissue expression (and possible disease modulation) are respectively: CerS1 in brain, skeletal muscle and testis which is responsible for synthesizes 18-carbon (C18) ceramide in brain (epilepsy, head/neck cancer); CerS2 which is responsible for the synthesis of the very-long-chain ceramides (VLC), e.g. C24, C24:1 in liver and kidney (breast cancer, neurological diseases); CerS3 which is involved in the synthesis of ceramides with ultra-long-chain acyl moieties 
(ULC-Cers) in skin and testis (epidermal defects); CerS4 that catalyzes production of Ceramides containing C18-22 fatty acids in skin, heart, and liver (breast cancer, neurological diseases); CerS5 which synthesizes C16-ceramide; a main proapoptotic ceramide; in lung epithelium (leukemia, colorectal cancer); and CerS6 which preferentially generates C14- and C16-ceramides in intestine, spleen, lymph nodes and thymus (breast cancer, neurological diseases, colitis) [5, 10-16].Sphingomyelin hydrolysis is another pathway through which ceramide is generated; sphingomyelin is converted to ceramide by sphingomyelinase enzyme activity [17].

Ceramide is also produced via the salvage pathway. In this case, sphingosine is produced as the product of sphingolipid catabolism, and is mostly salvaged through re-acylation, resulting in ceramide production [18].

Overall, in physiological conditions, ceramides provide structural integrity and act in a variety of pathways, including the heat shock response, apoptosis, cell cycle arrest, inflammation, oxidative stress, and modulation of gene expression [4, 5]. Many of the underlying mechanisms of ceramide action are still poorly understood, and probably include inhibition of the activity of mitogen-activated protein-kinases (MAPKs), activation of stress-activated protein-kinases (SAPKs) and apoptotic mithocondrial pathways, mimicking of the cytotoxic effects of many cytokines, and stimulation of pro-inflammatory adipokine secretion $[19,20]$. The accumulation of ceramide in sphingolipid-rich domains (lipid rafts) and their coalescence may affect signaling by clustering of receptors and signaling molecules [19]. Indeed, the sphingomyelinase enzymes are activated by many cytokines, including tumor necrosis factor$\alpha($ TNF- $\alpha$ ), interferon- $\gamma$ (IFN- $\gamma)$, interleukins (IL), such as IL-1 $\beta$, or platelet-activating factor (PAF) and adiponectin [21]. Besides inflammatory signals, oxidative stress increases ceramide production as well [22]. 
Furthermore, ceramides have been reported as potential biomarkers of many pathological diseases and conditions. Herein, their hypothesized role in the pathogenesis of diabetes mellitus and its chronic complications is described.

\section{Ceramide and diabetes mellitus, is there any verified relationship?}

In addition to primary beta cell dysfunction as an underlying cause, type 2 diabetes mellitus is well recognized as a metabolic disorder in which insulin resistance in peripheral cells is a central feature. Insulin resistance interferes with cellular consumption of glucose as a first-order substrate for metabolism and ATP (adenosine triphosphate) generation [23]. Whilst it has been purported that insulin resistance serves as a physiological defense against metabolic stress and overfeeding, it is also recognized as a hallmark of disease in many patients with type 2 diabetes [24].

The exact pathophysiology of insulin resistance is as yet unknown, but strong evidence points to the involvement and interplay of a variety of factors: a defect in GLUT4 (Glucose transporter type 4) expression, a defect in muscle glucose transport, impaired muscle glycogen synthesis, deviations in insulin receptor isoform expression or insulin/IGF-I hybrid receptor abundance and defects in the insulin signaling pathway. One example of the latter is the IR/IRS-1/PI 3-kinase/PKCz/l/Akt/GLUT4 cascade, where any defect in this pathway may contribute to the development of insulin resistance [25]. A body of evidence also suggests that other factors, such as high fat diet, overfeeding, inflammatory cytokines, free radical species and insulin signaling inhibitors, can induce insulin resistance and, ultimately, in the presence of beta-cell dysfunction, type 2 diabetes mellitus [26].

More recent work has suggested a causal relationship between ceramide production and beta cell dysfunction and insulin resistance [27]. It has been suggested that ceramide may play a role in beta cell apoptosis, pancreatic inflammation, mitochondrial stress, endoplasmic reticulum (ER) stress, adipokine release, IRS-1 (Insulin receptor substrate 1) phosphorylation, 
oxidative stress and insulin synthesis [6]. The evidence supporting the involvement of ceramide in each of these molecular pathways, known to be involved in the development of diabetes mellitus, is discussed in greater detail below, by distinguishing those mechanisms more specifically correlated with insulin resistance from those mainly implicated in beta-cell dysfunction.

\section{Ceramide and beta cell dysfunction}

\section{Ceramide and pancreatic beta cell apoptosis}

Apoptotic death of beta cells residing in pancreatic islets is a hallmark of pancreatic dysfunction in diabetic subjects. Loss of beta cell mass reduces the intrinsic capacity for insulin production, secretion and, therefore, release [28]. Apoptosis of beta cells in islets is a feature of both type 1 and type 2 diabetes. Beta cells are affected by metabolic factors that induce protein misfolding and/or induce inflammatory responses [28]. Loss of beta cells through apoptosis reduces the total effective beta cell mass and results in inadequate secreted insulin in response to a glucose load [29]. Beta cells are sensitive to a variety of pro-apoptotic stimuli, including ceramide [30].

Various clinical and experimental studies have demonstrated that sphingolipid metabolites, such as sphingosine 1-phosphate, glycosphingolipids and ceramide, can induce signaling pathways involved in beta cell apoptosis [31]. This can be accomplished either by inducing ER stress, enhancing lipotoxicity or disrupting mitochondrial function with subsequent development of oxidative damage [28]. Grösch and coworkers found that ceramide can stimulate cytochrome-C release and trigger down-stream apoptotic related pathways in beta cells [32]. Further, the apoptotic potency of ceramide was reported by Zhang et al in 2009, where they suggested that ceramide induces beta cell apoptosis through inhibition of ion channel activity [33]. Thus, induction of beta cell apoptosis is a direct diabetogenic consequence of high circulating plasma levels of ceramide. 


\section{Ceramide and pancreatic cell inflammation}

Pancreatic inflammation is strongly correlated to beta cell dysfunction and, accordingly, inadequate insulin secretion [34]. Much work has demonstrated that upregulation of cytokines and marked inflammatory responses are induced in an animal model of type 1 diabetes mellitus [35]. Major and colleagues in 1999 demonstrated that ceramide can mimic the cytotoxic effects of tumor necrosis factor- $\alpha$ (TNF- $\alpha$ ), interleukin-1 $\beta$ (IL-1 $\beta)$ and interferon- $\gamma$ (IFN- $\gamma$ ) cytokines in pancreatic beta cells and trigger related inflammatory responses [36]. Sjöholm et al. in 1995 also found that ceramide mimics IL-1 $\beta$ activity and promotes its cytotoxic and cytostatic influence in islet beta cells [37]. Moreover, Gregory et al recently reported the same effects of sphingolipids and found that they are involved in chronic inflammation in different tissues, including pancreatic islets [38]. Consequently, induction of inflammation is another possible effect of ceramide in diabetes development.

\section{Ceramide and pancreatic oxidative stress}

Oxidative stress has a pivotal role in the pathophysiology of many diseases, including the development of diabetes consequent upon beta cell stress [39]. Excess free radicals in beta cells, due to hyperglycemia or toxicity, can impair insulin gene expression and insulin secretion, and induce beta cells apoptosis [40]. Ceramide production enhances free radical generation and oxidative damage [41]. High levels of circulating ceramides are associated with mitochondrial dysfunction, thereby resulting in further free radical generation [42]. Czubowicz et al in 2014 showed that ceramide induces poly (ADP-ribose) polymerase-1 (PARP-1) activity, free radical generation and oxidative damage in neuronal cells [42]. While no direct evidence shows ceramide-induced oxidative damage in beta cells, indirect evidence indicates that oxidative stress due to ceramide accumulation can play a significant role in islet 
dysfunction, impaired insulin secretion and diabetes development, and this relationship clearly requires further investigation.

\section{Ceramide and defects in insulin expression}

Some studies have proposed that insulin mRNA/gene expression is under the influence of plasma levels of ceramide [43]. A number of different sphingolipid metabolites, in addition to ceramide, are strongly implicated in insulin gene expression and may exert inhibitory effects

upon it [31]. Kelpe et al in 2003 demonstrated that prolonged exposure to higher levels of ceramide down-regulates insulin gene expression in a model of isolated islets in culture [43]. Guo and coworkers in 2010 demonstrated that accumulation of ceramide in plasma inhibits proinsulin gene expression through protein phosphatase 2A (PP2A) activity [44]. Therefore, we conclude that ceramide-induced insulin gene down-regulation can be considered as another potential effect of ceramide in diabetes development.

\section{Ceramide and defects in insulin secretion}

Reduced insulin secretion is a central element in the pathogenesis of diabetes mellitus which may be due to lower beta cell mass or failure of the beta cell secretory machinery [45]. Free fatty acids, as well as ceramide, can affect insulin secretion in different ways, including via intracellular trafficking, intracellular storage in Golgi or by inhibiting secretory machinery [31]. Ceramide disrupts the apparatus transporting insulin from ER to Golgi and so disrupts intracellular trafficking of insulin and its subsequent secretion from beta cells [31]. Karunakaran et al in 2015 reported that ceramide accumulation is involved in both beta cell failure and disturbed insulin secretion [46]. Lang et al in 2011 reported that any defect in sphingomyelin synthase 1 activity, which naturally converts ceramide to sphingomyelin, leads to accumulation of ceramide and impairment of insulin release [20]. Therefore, it can logically 
be concluded that impairment of the insulin secretion machinery is another mechanism by which ceramide may induce diabetes mellitus.

\section{Ceramide and insulin resistance}

\section{Ceramide and glucose metabolism}

As stated earlier, insulin resistance is a hallmark of type 2 diabetes mellitus in which the beta cells are unable to secrete adequate levels of insulin into the circulation to overcome the resistance to this hormone; therefore, the entry of glucose into cells for use as a first line substrate is disturbed [47]. Any agent that interferes with the insulin signaling pathway can therefore induce insulin resistance and, ultimately, in the presence of beta-cell failure lead to type 2 diabetes mellitus [47]. Available evidence suggests that higher levels of plasma ceramides promote insulin resistance and the development of the metabolic syndrome [48]. Additionally, pharmacological or genetic disruption of ceramide synthesis has been shown to improve glucose metabolism and enhance insulin sensitization [48].

Haus and coworkers in 2009 showed that the plasma level of ceramide is directly associated with insulin resistance in people with type 2 diabetes and obesity [49]. They found that insulin sensitivity in those patients is inversely correlated to the ceramide species C18:0, C20:0, C24:1 and to the total plasma level of ceramide [49]. Recently, Cimmino et at demonstrated that the Czcer species of ceramide antagonizes insulin-signaling and induces insulin resistance [50]. The accumulation of ceramide produced from palmitate (through the de novo pathway) has been found to induce an inhibition of insulin signaling (in vitro) and diabetes (in vivo) [51, 52]. Chavez et al in 2012 also emphasized the role of ceramide in insulin resistance and presented a new hypothesis that implicated ceramide as a key agent in the development of insulin resistance [27]. 
Another possible mechanism for ceramide-induced insulin resistance is via insulin receptor substrate-1 (IRS-1) phosphorylation [53]. IRS-1 is a mediator protein with signaling activity that links the binding of insulin and insulin like growth factor-1 (IGF-1) to related intracellular receptors of the insulin pathways, thus triggering associated down-stream signaling pathways [54]. IRS-1 has approximately 232 Ser/Thr residues which are mainly subjected to phosphorylation [55]. Previous work has shown that Ser/Thr residue phosphorylation of IRS-1 decreases its ability to respond to insulin and negatively modulates insulin signaling pathways, thus contributing to insulin resistance $[54,55]$. Other reports indicate that ceramides may be directly involved in IRS-1 phosphorylation and can therefore negatively modulate insulin signaling and induce insulin resistance $[53,55]$. Ceramide inhibits IRS1 through activation of the PKR/JNK axis [56].

The accumulation of ceramide via different mechanisms blocks the activation of the Akt pathway, a protein kinase that is essential for insulin signaling and insulin-mediated stimulation of glucose metabolism, with consequent inhibition of the Akt-mediated GLUT4 translocation and glucose uptake, and glycogen synthesis [56-58]. Intriguingly, ceramides were found to play a role in insulin resistance induced by saturated fatty acids [59]. It has also been shown that ceramides can impair glucose metabolism by acting on Akt signaling pathways through PP2A and/or PKCל (zeta) mediators [60]. Chen et al. demonstrated that ceramides impair glucose metabolism and induce insulin resistance through PP2A- and PKC -dependent mechanisms [60]. Also, Mahfouz and coworkers found that ceramides induces insulin resistance through a PP2A-dependent pathway in skeletal muscles of animals [61].

Insulin resistance in skeletal muscle plays a key role in type 2 diabetes development. Different studies support the importance of specific muscle ceramides in the development of lipid-induced skeletal muscle insulin resistance [62, 63]. On the other hand, the role of ceramides as mediators of hepatic insulin resistance is unclear and supported by a few $[7,64$, 
65], but not all [66] studies, as recently reviewed [67]. The degradation of ceramides both in the liver and in fat tissue improved glucose metabolism, thus suggesting a major contributing role for both these tissues in regulating circulating ceramide levels, and, in addition, confirming the role of these substances on glucose homeostasis [7].

\section{Ceramide and ER stress}

Endoplasmic reticulum (ER) stress is defined as any disturbance in proper function of ER which may be due to improper folding or ER overloading [68]. ER stress is a prominent marker of insulin-resistant cells which, in turn, further impairs glucose homeostasis [69]. Ceramide can induce ER stress and impair glucose homeostasis in a number of experimental models in several cell lines such as islets beta cells [69, 70]. For example, Liu et al in 2014 found that exogenous application of ceramide induces severe ER stress by impairing ER $\mathrm{Ca}^{2+}$ homeostasis in human adenoid cystic carcinoma cells [70]. Yano and colleagues in 2011 showed that ceramide can cause ER stress by inducing protein misfolding in ER due to $\mathrm{Ca}^{2+}$ depletion in pancreatic $\beta$-cells [71]. Moreover, de la Monte and coworkers in 2012 demonstrated that ceramide-induced ER stress impaired the IGF (Insulin-like growth factor) signaling pathway and enhanced insulin resistance in postmortem brain tissue of human Alzheimer disease models [72].

However, specifically in muscle cells, ceramide-induced insulin resistance is mediated mainly by impairment of the insulin signaling pathway (IRS1 and Akt) [73], rather than by ceramideinduced ER stress, which probably plays a major role in the liver and adipose tissue [74].

\section{Ceramide and Adipokines Expression/Release}

The adipokines, also known as adipocytokines, are adipose tissue driven cytokines with pro-inflammatory properties that are mainly released by adipocytes. Since the recognition of 
leptin as the first adipokine, hundreds of these cytokines have been discovered, such as resistin, TNF- $\alpha$ and interleukin-6; this effectively implies that adipose tissue is a major endocrine organ [75]. Adipokines can exert diabetogenic effects by enhancing insulin resistance in peripheral tissues of subjects with obesity [76]. Although some adipokines, such as leptin and adiponectin, are involved in control of feeding behavior and balance of energy, more recent evidence confirms that they are also involved in inflammatory response-induced insulin resistance [76].

Some evidence suggests that ceramide increases adipokine expression and increases its level in the circulation [48]. Available data indicate a strong link between ceramide and adipokines [48, 77]. Hamada and colleagues in 2014 demonstrated that ceramide stimulates proinflammatory adipokine secretion as well as IL-6 and monocyte chemo-attractant protein-1 (MCP-1) in mature 3T3-L1 adipocytes [77]. In addition, Chavez et al in 2012 showed that ceramide production is directly correlated to leptin, TNF $\alpha$, adiponectin and resistin levels in tissues [27]. Furthermore, the adiponectin receptor is linked to ceramidase activity and induced activation of this activity enhances ceramide catabolism, formation of its antiapoptotic metabolite--sphingosine-1-phosphate, and subsequent decline in tissue ceramide levels, thus contributing to the pleiotropic metabolic actions of adiponectin [78].

Therefore, ceramide-induced adipokine secretion can be considered as another pathophysiologic pathway involved in insulin resistance and type 2 diabetes mellitus development.

\section{Ceramide and obesity}

People with obesity are often insulin resistant and show an increased risk of developing type 2 diabetes mellitus. Plasma ceramide concentrations are increased in people with obesity (41). Obesity leads to increased circulating substrates required for de novo ceramide synthesis 
(palmitate and amino acids) [79]. Furthermore, the reduced blood adiponectin levels associated with obesity, with the resultant reduction in adiponectin-receptor mediated ceramidase activity [78], together with the increased sphingomyelinase activity induced by increased circulating TNF- $\alpha$ concentrations, are characteristic of people with obesity [80] and lead to tissue ceramide accumulation. Specific muscle and adipose ceramide compounds were found to be inversely associated with energy expenditure and predictive of weight gain [81].

A relationship between ceramides and pro-inflammatory cytokines may be implicated in both reduced insulin sensitivity and fat accumulation. In particular, ectopic fat accumulation characterizes obesity and is critical in the development of obesity-associated diseases. Ceramides are among the lipid metabolites accumulating in ectopic sites, such as the liver, skeletal muscle, and heart [80]. A high-fat diet-induced both insulin resistance and a distinctive ceramide accumulation in fat, liver and muscle tissues in mice [82]. By contrast, weight loss by bariatric surgery plus exercise reduced muscle ceramides and improved insulin sensitivity [83].

From this evidence, we can conclude that ceramides, through their metabolic effects and tissue accumulation, contribute to insulin resistance, conferring an increased risk for development of diabetes.

\section{Roles of ceramides in chronic complications of diabetes mellitus}

Ceramides, other than in pancreas, adipose tissue, muscle, liver, and brain, may accumulate in tissues such as kidney, eye, heart and vessels, thus contributing to chronic diabetes complications.

Increased ceramides and specific ceramide ratios have been reported to be associated with an increased risk of death and negative outcomes in patients with cardiovascular diseases [84-86]. Ceramides may contribute causally to impaired vasoreactivity, dyslipidemia, aggregation of 
lipoproteins, uptake and retention of oxidized lipids in the vascular wall, and, ultimately, to atherosclerotic plaque formation [67, 80]. Ceramide has been associated with lipotoxic and diabetic cardiomyopathy in animal models [87, 88].

Furthermore, these substances have been implicated in mesangial cell apoptosis [89] and in retinal pericyte apoptosis [90] which are associated with diabetic nephropathy and retinal pericyte apoptosis, respectively

Therefore, tissue accumulation of ceramides may not only have a pathogenic, but also a prognostic significance in diabetes mellitus, being associated with the risk of development of diabetes-related complications.

\section{Clinical implications}

An increasing amount of evidence highlights the role of ceramide as a pathogenetic factor especially in type 2 diabetes mellitus. However whether ceramide disease modulation occurs as a response to pathological mechanisms, such as inflammation or cellular stress, or is actively involved in diabetes pathogenesis, is still unclear.

Ceramides can be detected in blood samples and have been proposed as disease biomarker [86]. Wigger et al in 2017 reported that plasma dihydroceramides are important predictors of diabetes and confirmed in two cohort studies that dihydroceramides are increase by up to 9 years before the onset of diabetes in suspected subjects [91]. In particular, increased plasma concentrations of ceramides C18:0, C20:0, C24:1 have been found in patients with type 2 diabetes mellitus compared to control subjects [53], and plasma C18:0, C20:0, C22:0 and some dihydroceramide species were significantly elevated in patient plasma many years prior to diabetes diagnosis [5]. A recent large prospective study confirmed the significant and independent role of ceramides (in particular, the Cer(18:1/18:0)/Cer(18:1/16:0) ceramide ratio) in the prediction of type 2 diabetes up to 10 years before the onset of the disease [92]. 
Furthermore, specific ceramides are associated early with insulin resistance, prior to its progression to diabetes [93]. This is intriguing given the fact that the early stages of diabetes are usually asymptomatic.

Ceramide ratios were improved following lifestyle intervention that included weight loss [92]. Intriguingly, the possibility of reducing circulating levels of specific ceramides by increasing fruit and vegetable consumption has been recently reported [94]. Therefore, specific ceramides or ceramides ratio might serve as useful biomarkers for diabetes risk, and can possibly be modulated by reductions in body mass index and a healthier lifestyle.

Similarly, the use of ceramides might be useful in the evaluation of cardiovascular risk and identification of patients requiring more aggressive treatment [95]. This is a particularly important assessment in people with diabetes, since cardiovascular diseases are the leading cause of their premature mortality.

Finally, the possibility of developing pharmacological ceramide antagonists that exert their effect via downregulation of the enzymes implicated in ceramide synthesis, offers an interesting future perspective in protection from, and treatment of, diabetes, obesity and cardiovascular diseases. Whilst all the available evidence mechanistically implicates ceramides in glucose/insulin metabolism, diabetes mellitus and its complications, randomized controlled human trials are needed to conclude this definitively.

\section{Conclusion}

Ceramides are lipid molecules, primarily located in eukaryotic cell membranes, which are integral to the lipid bilayer as well as many intracellular signaling pathways. Recent evidence suggests that they also exert pathological effects and, as such, they may be causally involved in the development of diabetes mellitus. Based on our review of the available data, we conclude that ceramides can interfere with glucose homeostasis, induce insulin resistance 
and enhance the likelihood of diabetes development; these effects are mediated through at least eight molecular pathways. Development of new therapeutic agents that allow pharmacological lowering of ceramide synthesis by using ceramide synthase inhibitors which precisely suppress ceramide production, and therefore plasma levels, may be beneficial in prevention and/or treatment of diabetes and its related complications. Since ceramides have regular tissue expression, using ceramide inhibitors may provide new therapeutic targets against diabetes and its complications.

\section{Conflict of Interests}

The authors declare no conflict of interest.

\section{Acknowledgment}

The authors are thankful to the "Clinical Research and Development Unit" of the Baqiyatallah Hospital (Tehran, Iran) for providing technical support.

\section{Funding and support}

The authors declare no funding support by any organization or university for this study. 


\section{References}

1. Jansson, S., et al., Prevalence and incidence of diabetes mellitus: a nationwide populationbased pharmaco-epidemiological study in Sweden. Diabetic Medicine, 2015. 32(10): p. 13191328.

2. Navarro-González, J.F., et al., Inflammatory molecules and pathways in the pathogenesis of diabetic nephropathy. Nature Reviews Nephrology, 2011. 7(6): p. 327.

3. Kummarapurugu, A., et al., Neutrophil Elastase And Ceramide: Impact On Cystic Fibrosis Lung Inflammation. Am J Respir Crit Care Med, 2017. 195: p. A1298.

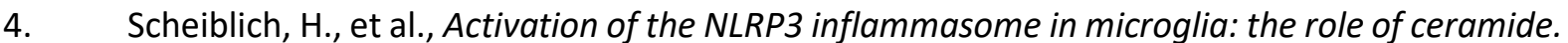
Journal of neurochemistry, 2017. 143(5): p. 534-550.

5. Kurz, J., et al., Ceramides as Novel Disease Biomarkers. Trends in molecular medicine, 2019. 25(1): p.20-32.

6. Galadari, S., et al., Role of ceramide in diabetes mellitus: evidence and mechanisms. Lipids in health and disease, 2013. 12(1): p. 98.

7. Xia, J.Y., et al., Targeted induction of ceramide degradation leads to improved systemic metabolism and reduced hepatic steatosis. Cell metabolism, 2015. 22(2): p. 266-278.

8. Rabionet, M., K. Gorgas, and R. Sandhoff, Ceramide synthesis in the epidermis. Biochimica et Biophysica Acta (BBA)-Molecular and Cell Biology of Lipids, 2014. 1841(3): p. 422-434.

9. Rodriguez-Cuenca, S., N. Barbarroja, and A. Vidal-Puig, Dihydroceramide desaturase 1, the gatekeeper of ceramide induced lipotoxicity. Biochimica et Biophysica Acta (BBA)-Molecular and Cell Biology of Lipids, 2015. 1851(1): p. 40-50.

10. Mullen, T.D., Y.A. Hannun, and L.M. Obeid, Ceramide synthases at the centre of sphingolipid metabolism and biology. Biochemical Journal, 2012. 441(3): p. 789-802.

11. Turner, N., et al., A selective inhibitor of ceramide synthase 1 reveals a novel role in fat metabolism. Nature communications, 2018. 9(1): p. 3165.

12. Spassieva, S.D., et al., Disruption of ceramide synthesis by CerS2 down-regulation leads to autophagy and the unfolded protein response. Biochemical Journal, 2009. 424(2): p. 273-283.

13. Radner, F.P., et al., Mutations in CERS3 cause autosomal recessive congenital ichthyosis in humans. PLoS genetics, 2013. 9(6): p. e1003536.

14. Laviad, E.L., et al., Characterization of Ceramide Synthase 2 Tissue distribution, substrate specificity, and inhibition by sphingosine 1-phosphate. Journal of Biological Chemistry, 2008. 283(9): p. 5677-5684.

15. Lahiri, S. and A.H. Futerman, LASS5 is a bona fide dihydroceramide synthase that selectively utilizes palmitoyl-CoA as acyl donor. Journal of Biological Chemistry, 2005. 280(40): p. 3373533738.

16. Tirodkar, T.S., et al., Expression of ceramide synthase 6 transcriptionally activates acid ceramidase in a c-Jun N-terminal Kinase (JNK)-dependent manner. Journal of Biological Chemistry, 2015: p. jbc. M114. 631325.

17. Hannun, Y.A. and B. Newcomb, A new twist to the emerging functions of ceramides in cancer: novel role for platelet acid sphingomyelinase in cancer metastasis. EMBO molecular medicine, 2015. 7(6): p. 692-694.

18. Kitatani, K., J. Idkowiak-Baldys, and Y.A. Hannun, The sphingolipid salvage pathway in ceramide metabolism and signaling. Cellular signalling, 2008. 20(6): p. 1010-1018.

19. Woodcock, J., Sphingosine and ceramide signalling in apoptosis. IUBMB life, 2006. 58(8): p. 462-466.

20. Lang, F., S. Ullrich, and E. Gulbins, Ceramide formation as a target in beta-cell survival and function. Expert opinion on therapeutic targets, 2011. 15(9): p. 1061-1071.

21. Gomez-Munoz, A., et al., Control of inflammatory responses by ceramide, sphingosine 1phosphate and ceramide 1-phosphate. Progress in lipid research, 2016. 61: p. 51-62. 
22. Zeidan, Y. and Y. Hannun, The acid sphingomyelinase/ceramide pathway: biomedical significance and mechanisms of regulation. Current molecular medicine, 2010. 10(5): p. 454466.

23. Altaf, Q.A., A. Barnett, and A. Tahrani, Novel therapeutics for type 2 diabetes: insulin resistance. Diabetes, Obesity and Metabolism, 2015. 17(4): p. 319-334.

24. Arnold, S.E., et al., Brain insulin resistance in type 2 diabetes and Alzheimer disease: concepts and conundrums. Nature Reviews Neurology, 2018.

25. Sesti, G., Pathophysiology of insulin resistance. Best practice \& research Clinical endocrinology \& metabolism, 2006. 20(4): p. 665-679.

26. Hulston, C.J., A.A. Churnside, and M.C. Venables, Probiotic supplementation prevents high-fat, overfeeding-induced insulin resistance in human subjects. British Journal of Nutrition, 2015. 113(4): p. 596-602.

27. Chavez, J.A. and S.A. Summers, A ceramide-centric view of insulin resistance. Cell metabolism, 2012. 15(5): p. 585-594.

28. Thomas, H.E., et al., Beta cell apoptosis in diabetes. Apoptosis, 2009. 14(12): p. 1389.

29. Pick, A., et al., Role of apoptosis in failure of beta-cell mass compensation for insulin resistance and beta-cell defects in the male Zucker diabetic fatty rat. Diabetes, 1998. 47(3): p. 358-364.

30. Lupi, R., et al., Prolonged exposure to free fatty acids has cytostatic and pro-apoptotic effects on human pancreatic islets: evidence that b-cell death is caspase mediated, partially dependent on ceramide pathway, and Bcl-2 regulated. Diabetes, 2002. 51(5): p. 1437-1442.

31. Boslem, E., P.J. Meikle, and T.J. Biden, Roles of ceramide and sphingolipids in pancreatic B-cell function and dysfunction. Islets, 2012. 4(3): p. 177-187.

32. Grösch, S., S. Schiffmann, and G. Geisslinger, Chain length-specific properties of ceramides. Progress in lipid research, 2012. 51(1): p. 50-62.

33. Zhang, Y., et al., Sphingomyelinase dependent apoptosis following treatment of pancreatic beta-cells with amyloid peptides Aß1-42 or IAPP. Apoptosis, 2009. 14(7): p. 878-889.

34. Gaglia, J.L., et al., Noninvasive mapping of pancreatic inflammation in recent-onset type-1 diabetes patients. Proceedings of the National Academy of Sciences, 2015. 112(7): p. 21392144.

35. Sun, J., et al., Pancreatic B-cells limit autoimmune diabetes via an immunoregulatory antimicrobial peptide expressed under the influence of the gut microbiota. Immunity, 2015. 43(2): p. 304-317.

36. Major, C.D., Z. Gao, and B.A. Wolf, Activation of the sphingomyelinase/ceramide signal transduction pathway in insulin-secreting beta-cells: role in cytokine-induced beta-cell death. Diabetes, 1999. 48(7): p. 1372-1380.

37. Sjöholm, A., Ceramide inhibits pancreatic $B$-cell insulin production and mitogenesis and mimics the actions of interleukin-16. FEBS letters, 1995. 367(3): p. 283-286.

38. Norris, G.H. and C.N. Blesso, Dietary and Endogenous Sphingolipid Metabolism in Chronic Inflammation. Nutrients, 2017. 9(11): p. 1180.

39. Martín, M.Á., et al., Cocoa flavonoid epicatechin protects pancreatic beta cell viability and function against oxidative stress. Molecular nutrition \& food research, 2014. 58(3): p. 447456.

40. Robertson, R.P., Chronic oxidative stress as a central mechanism for glucose toxicity in pancreatic islet beta cells in diabetes. Journal of Biological Chemistry, 2004. 279(41): p. 4235142354.

41. Sanvicens, N. and T.G. Cotter, Ceramide is the key mediator of oxidative stress-induced apoptosis in retinal photoreceptor cells. Journal of neurochemistry, 2006. 98(5): p. 1432-1444.

42. Jazvinšćak Jembrek, M., P.R. Hof, and G. Šimić, Ceramides in Alzheimer's disease: key mediators of neuronal apoptosis induced by oxidative stress and $A B$ accumulation. Oxidative medicine and cellular longevity, 2015. 2015. 
43. Kelpe, C.L., et al., Palmitate inhibition of insulin gene expression is mediated at the transcriptional level via ceramide synthesis. Journal of Biological Chemistry, 2003. 278(32): p. 30015-30021.

44. Guo, J., et al., Blockage of ceramide metabolism exacerbates palmitate inhibition of pro-insulin gene expression in pancreatic B-cells. Molecular and cellular biochemistry, 2010. 338(1-2): p. 283-290.

45. Tang, C., et al., Loss of FFA2 and FFA3 increases insulin secretion and improves glucose tolerance in type 2 diabetes. Nature medicine, 2015. 21(2): p. 173.

46. Karunakaran, U., et al., CD36 initiated signaling mediates ceramide-induced TXNIP expression in pancreatic beta-cells. Biochimica et Biophysica Acta (BBA)-Molecular Basis of Disease, 2015. 1852(11): p. 2414-2422.

47. Samuel, V.T. and G.I. Shulman, The pathogenesis of insulin resistance: integrating signaling pathways and substrate flux. The Journal of clinical investigation, 2016. 126(1): p. 12-22.

48. Xia, J.Y., T.S. Morley, and P.E. Scherer, The adipokine/ceramide axis: key aspects of insulin sensitization. Biochimie, 2014. 96: p. 130-139.

49. Haus, J.M., et al., Plasma ceramides are elevated in obese subjects with type 2 diabetes and correlate with the severity of insulin resistance. Diabetes, 2009. 58(2): p. 337-343.

50. Cimmino, I., et al., A peptide antagonist of Prep1-p160 interaction improves ceramide-induced insulin resistance in skeletal muscle cells. Oncotarget, 2017. 8(42): p. 71845.

51. Huang, S., et al., Downregulation of lipin-1 induces insulin resistance by increasing intracellular ceramide accumulation in C2C12 myotubes. International journal of biological sciences, 2017. 13(1): p. 1.

52. Chaurasia, B. and S.A. Summers, Ceramides-lipotoxic inducers of metabolic disorders. Trends in Endocrinology \& Metabolism, 2015. 26(10): p. 538-550.

53. Yu, C., et al., Mechanism by which fatty acids inhibit insulin activation of insulin receptor substrate-1 (IRS-1)-associated phosphatidylinositol 3-kinase activity in muscle. Journal of Biological Chemistry, 2002. 277(52): p. 50230-50236.

54. Gao, Z., et al., Serine phosphorylation of insulin receptor substrate 1 by inhibitor $K B$ kinase complex. Journal of Biological Chemistry, 2002. 277(50): p. 48115-48121.

55. Herschkovitz, A., et al., Common inhibitory serine sites phosphorylated by IRS-1 kinases, triggered by insulin and inducers of insulin resistance. Journal of Biological Chemistry, 2007. 282(25): p. 18018-18027.

56. Hassan, R.H., et al., Sustained Action of Ceramide on the Insulin Signaling Pathway in Muscle Cells IMPLICATION OF THE DOUBLE-STRANDED RNA-ACTIVATED PROTEIN KINASE. Journal of Biological Chemistry, 2016. 291(6): p. 3019-3029.

57. Summers, S.A., et al., Regulation of insulin-stimulated glucose transporter GLUT4 translocation and Akt kinase activity by ceramide. Molecular and cellular biology, 1998. 18(9): p. 5457-5464.

58. Hajduch, E., et al., Ceramide impairs the insulin-dependent membrane recruitment of protein kinase B leading to a loss in downstream signalling in L6 skeletal muscle cells. Diabetologia, 2001. 44(2): p. 173-183.

59. Holland, W.L., et al., Inhibition of ceramide synthesis ameliorates glucocorticoid-, saturatedfat-, and obesity-induced insulin resistance. Cell metabolism, 2007. 5(3): p. 167-179.

60. Chen, T.-C., et al., The glucocorticoid-Angpt/4-ceramide axis induces insulin resistance through PP2A and PKCל. Sci. Signal., 2017. 10(489): p. eaai7905.

61. Mahfouz, R., et al., Characterising the inhibitory actions of ceramide upon insulin signaling in different skeletal muscle cell models: a mechanistic insight. PLoS One, 2014. 9(7): p. e101865.

62. Tonks, K.T., et al., Skeletal muscle and plasma lipidomic signatures of insulin resistance and overweight/obesity in humans. Obesity, 2016. 24(4): p. 908-916. 
63. Summers, S.A. and B.H. Goodpaster, CrossTalk proposal: Intramyocellular ceramide accumulation does modulate insulin resistance. The Journal of physiology, 2016. 594(12): p. 3167-3170.

64. Luukkonen, P.K., et al., Hepatic ceramides dissociate steatosis and insulin resistance in patients with non-alcoholic fatty liver disease. Journal of hepatology, 2016. 64(5): p. 1167-1175.

65. Wasilewska, N., et al., Increased serum concentration of ceramides in obese children with nonalcoholic fatty liver disease. Lipids in health and disease, 2018. 17(1): p. 216.

66. Ter Horst, K.W., et al., Hepatic diacylglycerol-associated protein kinase CE translocation links hepatic steatosis to hepatic insulin resistance in humans. Cell reports, 2017. 19(10): p. 19972004.

67. Petersen, M.C. and G.I. Shulman, Roles of diacylglycerols and ceramides in hepatic insulin resistance. Trends in pharmacological sciences, 2017. 38(7): p. 649-665.

68. Mechanistically, B.E.R.S., Endoplasmic Reticulum Stress. Hypertension, 2015.

69. Rajan, S.S., et al., Endoplasmic reticulum (ER) stress \& diabetes. Indian Journal of Medical Research, 2007. 125(3): p. 411.

70. Liu, Z., et al., Induction of ER stress-mediated apoptosis by ceramide via disruption of ER Ca 2+ homeostasis in human adenoid cystic carcinoma cells. Cell \& bioscience, 2014. 4(1): p. 71.

71. Yano, M., et al., Mitochondrial dysfunction and increased reactive oxygen species impair insulin secretion in sphingomyelin synthase 1-null mice. Journal of Biological Chemistry, 2011. 286(5): p. 3992-4002.

72. de la Monte, S.M., et al., Dysfunctional pro-ceramide, ER stress, and insulin/IGF signaling networks with progression of Alzheimer's disease. Journal of Alzheimer's Disease, 2012. 30(s2): p. S217-S229.

73. Hassan, R.H., et al., Endoplasmic reticulum stress does not mediate palmitate-induced insulin resistance in mouse and human muscle cells. Diabetologia, 2012. 55(1): p. 204-214.

74. Rieusset, J., et al., Reduction of endoplasmic reticulum stress using chemical chaperones or Grp78 overexpression does not protect muscle cells from palmitate-induced insulin resistance. Biochemical and biophysical research communications, 2012. 417(1): p. 439-445.

75. Lehr, S., S. Hartwig, and H. Sell, Adipokines: a treasure trove for the discovery of biomarkers for metabolic disorders. PROTEOMICS-Clinical Applications, 2012. 6(1-2): p. 91-101.

76. Pessin, J.E. and H. Kwon, Adipokines mediate inflammation and insulin resistance. Frontiers in endocrinology, 2013. 4: p. 71.

77. Hamada, Y., et al., Involvement of de novo ceramide synthesis in pro-inflammatory adipokine secretion and adipocyte-macrophage interaction. The Journal of nutritional biochemistry, 2014. 25(12): p. 1309-1316.

78. Holland, W.L., et al., Receptor-mediated activation of ceramidase activity initiates the pleiotropic actions of adiponectin. Nature medicine, 2011. 17(1): p. 55.

79. Newgard, C.B., et al., A branched-chain amino acid-related metabolic signature that differentiates obese and lean humans and contributes to insulin resistance. Cell metabolism, 2009. 9(4): p. 311-326.

80. Aburasayn, H., R.B. Al, and J.R. Ussher, Targeting ceramide metabolism in obesity. American journal of physiology. Endocrinology and metabolism, 2016. 311(2): p. E423-35.

81. Heinitz, S., et al., Specific skeletal muscle sphingolipid compounds in energy expenditure regulation and weight gain in Native Americans of Southwestern heritage. International Journal of Obesity, 2017. 41(10): p. 1585.

82. Turner, N., et al., Distinct patterns of tissue-specific lipid accumulation during the induction of insulin resistance in mice by high-fat feeding. Diabetologia, 2013. 56(7): p. 1638-1648.

83. Neunhaeuserer, D., et al., Functional Evaluation in Obese Patients Before and After Sleeve Gastrectomy. Obesity surgery, 2017. 27(12): p. 3230-3239.

84. Jiang, X.-c., et al., Plasma sphingomyelin level as a risk factor for coronary artery disease. Arteriosclerosis, thrombosis, and vascular biology, 2000. 20(12): p. 2614-2618. 
85. Laaksonen, R., et al., Plasma ceramides predict cardiovascular death in patients with stable coronary artery disease and acute coronary syndromes beyond LDL-cholesterol. European heart journal, 2016. 37(25): p. 1967-1976.

86. Meeusen, J.W., et al., Plasma Ceramides: A Novel Predictor of Major Adverse Cardiovascular Events After Coronary Angiography. Arteriosclerosis, thrombosis, and vascular biology, 2018: p. ATVBAHA. 118.311199.

87. Park, T.-S., et al., Ceramide is a cardiotoxin in lipotoxic cardiomyopathy. Journal of lipid research, 2008. 49(10): p. 2101-2112.

88. Basu, R., et al., Type 1 diabetic cardiomyopathy in the Akita (Ins2 WT/C96Y) mouse model is characterized by lipotoxicity and diastolic dysfunction with preserved systolic function. American Journal of Physiology-Heart and Circulatory Physiology, 2009. 297(6): p. H2096H2108.

89. Liu, G., et al., Evaluation of sphingolipid metabolism in renal cortex of rats with streptozotocininduced diabetes and the effects of rapamycin. Nephrology Dialysis Transplantation, 2010. 26(5): p. 1493-1502.

90. Cacicedo, J.M., et al., Palmitate-induced apoptosis in cultured bovine retinal pericytes: roles of NAD (P) H oxidase, oxidant stress, and ceramide. Diabetes, 2005. 54(6): p. 1838-1845.

91. Wigger, L., et al., Plasma dihydroceramides are diabetes susceptibility biomarker candidates in mice and humans. Cell reports, 2017. 18(9): p. 2269-2279.

92. Hilvo, M., et al., Ceramide stearic to palmitic acid ratio predicts incident diabetes. Diabetologia, 2018. 61(6): p. 1424-1434.

93. Lemaitre, R.N., et al., Circulating Sphingolipids, Insulin, HOMA-IR and HOMA-B: the Strong Heart Family Study. Diabetes, 2018: p. db171449.

94. Mölsä, M., Rapid identification of selected pathogens in biothreat preparedness. Series 1: Research Publications No. 8, 2016.

95. Cui, S., et al., Plasma phospholipids and sphingolipids identify stent restenosis after percutaneous coronary intervention. JACC: Cardiovascular Interventions, 2017: p. 3163. 


\section{Figure legends}

Figure 1: the structure of a ceramide molecule, composed of sphingosine (upper half) and fatty acid (lower half)

Figure 2: Schematic delineating three known pathways of ceramide synthesis 